\title{
Hmga2 functions as a competing endogenous RNA to promote lung cancer progression
}

\author{
Madhu S. Kumar ${ }^{1}$, Elena Armenteros-Monterroso ${ }^{1}$, Philip East ${ }^{2}$, Probir Chakravorty ${ }^{2}$, Nik \\ Matthews $^{3}$, Monte M. Winslow ${ }^{4}$, and Julian Downward ${ }^{1,5}$ \\ ${ }^{1}$ Signal Transduction Laboratory, Cancer Research UK London Research Institute, 44 Lincoln's \\ Inn Fields, London WC2A 3LY, UK. \\ ${ }^{2}$ Bioinformatics and Biostatistics Group, Cancer Research UK London Research Institute, 44 \\ Lincoln's Inn Fields, London WC2A 3LY, UK. \\ ${ }^{3}$ Advanced Sequencing Facility, Cancer Research UK London Research Institute, 44 Lincoln's \\ Inn Fields, London WC2A 3LY, UK. \\ ${ }^{4}$ Department of Genetics, Department of Pathology, the Stanford Cancer Institute, Stanford \\ University School of Medicine, Stanford CA 94305, USA. \\ ${ }^{5}$ Lung Cancer Group, Division of Cancer Biology, The Institute of Cancer Research, 237 Fulham \\ Road, London SW3 6JB, UK.
}

\begin{abstract}
Non-small cell lung cancer (NSCLC) is the most prevalent histological cancer subtype worldwide $^{1}$. As the majority of patients present with invasive, metastatic disease ${ }^{2}$, it is vital to understand the basis for lung cancer progression. Hmga2 is highly expressed in metastatic lung adenocarcinoma where it contributes to cancer progression and metastasis ${ }^{3-6}$. Here we show that Hmga2 promotes lung cancer progression by operating as a competing endogenous RNA (ceRNA) ${ }^{7-11}$ for the let-7 microRNA (miRNA) family. Hmga2 can promote the transformation of lung cancer cells independent of protein-coding function but dependent upon the presence of let-7 sites; this occurs without changes in the levels of let-7 isoforms, suggesting that Hmga2 affects let-7 activity by altering miRNA targeting. These effects are further observed in vivo, where Hmga 2 ceRNA activity drives lung cancer growth, invasion and dissemination. Integrated analysis of miRNA target prediction algorithms and metastatic lung cancer gene expression data reveals the TGF- $\beta$ co-receptor $T g f b r 3^{12}$ as a putative target of $H m g a 2$ ceRNA function. $T g f b r 3$ expression is regulated by the Hmga 2 ceRNA via differential recruitment to Argonaute-2 (Ago2), and TGF- $\beta$ signalling driven by Tgfbr3 is largely necessary for Hmga2 to promote lung cancer progression. Finally, analysis of NSCLC patient gene expression data reveals that HMGA2 and TGFBR3 are co-ordinately regulated in NSCLC patient material, a vital corollary to ceRNA function. Taken together, these results suggest that Hmga2 promotes lung carcinogenesis as both a protein-coding gene and a non-coding RNA; such dual-function regulation of gene expression networks reflects a novel means by which oncogenes promote disease progression.
\end{abstract}

Correspondence and requests for materials should be addressed to J.D. (julian.downward@ cancer.org.uk)..

Author Contributions. M.S.K. and J.D. designed the study. M.S.K. and E.A.M. performed the experiments described. M.S.K., P.E., and P.C. conducted bioinformatics analyses. N.M. performed the next-generation sequencing studies. M.M.W. provided necessary reagents and conceptual advice. M.S.K. and J.D. wrote the manuscript.

Author Information: Reprints and permissions information is available at www.nature.com/reprints.

The authors declare no competing financial interests. 
The competing endogenous RNA hypothesis posits that specific RNAs can function as sinks for pools of active miRNAs, functionally liberating other transcripts targeted by that set of miRNAs ${ }^{10}$. Downregulation of the transcription factor $\mathrm{Nkx} 2.1$ promotes lung adenocarcinoma progression partially via derepression of $\mathrm{Hmga2}^{6}$, a non-histone chromosomal high mobility group protein. Intriguingly, Hmga2 has been described as a prototypic let-7 target transcript, with seven conserved sites in its $3^{\prime}$ untranslated region ( $3^{\prime}$ UTR ${ }^{13}$. Reduction of Hmga2 by RNA interference, which would deplete both Hmga2 protein and transcript, greatly reduces metastatic ability. Thus, it is possible that the transcript could operate independent of the protein in lung cancer progression.

To determine if Hmga 2 can operate as a ceRNA for the let-7 family, we generated an allelic series of Hmga2 expression constructs (Fig. 1a). In this series, we expressed the wild-type full-length Hmga2 cDNA (wt); Hmga2 with mutation of all seven predicted let-7 binding $\operatorname{sites}^{13}$ (m7); Hmga2 with mutation of the single in-frame start codon (ATG wt); or Hmga2 with mutation of both the start codon and the let-7 binding sites (ATG m7). We then examined these constructs in two lung cancer cell lines generated from the Kras $^{L S L-G 12 D}$; Trp53 floxfllox mouse model: a cell line derived from a non-metastatic lung tumour which expresses very low levels of Hmga2 (368T1); and a cell line derived from a lymph node metastasis which expresses high levels of $\operatorname{Hmga2}(482 \mathrm{~N} 1)^{6}$. Using two antibodies that recognize either the N-terminus or the second AT-hook of the protein (M. Narita, personal communication), we found that the Hmga2 wt and $\mathrm{m} 7$ constructs efficiently express full-length $\mathrm{Hmga} 2$ protein ( $\mathrm{m} 7$ over-expresses $\mathrm{Hmga} 2$ due to loss of let-7 mediated suppression) while the Hmga2 ATG wt and ATG m7 constructs do not (Fig. 1b).

Importantly, we observe similar levels of Hmga2 transcript expressed in the allelic series (in the case of the $482 \mathrm{~N} 1$ cell line, the allelic series was mutated to abrogate binding to a short hairpin RNA [shRNA] against Hmga2) (Fig. 1c). Moreover, expression of the allelic series has no effect on the expression of various let-7 family members (Extended Data Fig. 1a). Taken together, this Hmga2 allelic series allows us to compare specifically the roles of Hmga2 protein and transcript function on lung cell transformation.

We therefore compared the ability of the Hmga2 allelic series to promote anchorageindependent growth of the lung cancer cell lines. We observed a striking promotion of soft agar growth by both Hmga2 wt and ATG wt in the 368T1 and 482N1 cells (Figs. 1d and e); more modest growth was observed with Hmga2 m7, despite elevated protein expression relative to Hmga2 wt, and no growth was provided by Hmga2 ATG m7. This effect can be observed further in two additional human lung cancer cells (H1299 and SK-MES-1), as suppression of soft agar growth by HMGA2 depletion can be rescued robustly by Hmga2 wt and ATG wt but more modestly by Hmga $2 \mathrm{~m} 7$ (Extended Data Fig. 1b-e). Importantly, exogenous expression of let-7 reversed the ability of the Hmga 2 ceRNA to promote anchorage-independent growth, suggesting that let-7 regulates this effect (Extended Data Fig. 2b). To demonstrate that the effect of the Hmga 2 ceRNA is driven by let-7 sites in the $3^{\prime}$ untranslated region ( $\left.3^{\prime} \mathrm{UTR}\right)$, we expressed only the wild type or let-7 site mutated $3^{\prime}$ UTRs in 368T1 cells and examined the consequences on anchorage-independent growth. Strikingly, expression of the wild type but not let-7 mutant $3^{\prime}$ UTR was sufficient to promote soft agar growth in 368T1 cells (Extended Data Fig. 2c).

Beyond direct Hmga2 depletion, the Hmga2 wt and Hmga2 ATG wt constructs substantially rescued anchorage-independent growth in 482N1 cells stably over-expressing Nkx2.1, which we have previously shown to suppress lung cancer progression ${ }^{6}$ (Extended Data Fig. 2d). Notably, this effect is not due to a general proliferative benefit of Hmga2 wt and ATG wt cells, as BrdU incorporation in adherent conditions was comparable across the allelic series (Extended Data Fig. 3a). However, when lung cancer cells are placed in suspension, Hmga 2 depletion suppressed proliferation and this proliferation was rescued substantially by 
Hmga2 wt and ATG wt and more marginally by Hmga2 m7 (Extended Data Fig. 3b); in contrast, the rate of apoptosis in the allelic series was not affected by growth in suspension (data not shown). Taken together, these results suggest that the Hmga2 transcript functions in a largely protein coding-independent but let-7 site-dependent manner to promote lung cancer cell transformation in vitro.

To examine the effect of Hmga 2 ceRNA activity on lung cancer cell dissemination in vivo, we intravenously transplanted 482N1 cells expressing either the control shRNA and expression construct or the shRNA targeting Hmga2 plus the Hmga2 allelic series into syngeneic mice. As seen in Figure 2a, micro-CT analysis revealed a substantial rescue of lung tumour formation with expression of either the Hmga2 wt or ATG wt constructs (with more modest effects with the Hmga2 $\mathrm{m} 7$ construct). Histopathological analysis and quantification of surface lesions confirmed the effects of Hmga2 ceRNA activity on in vivo lung tumourigenesis (Figs. $2 \mathrm{~b}$ and c). Moreover, the control shRNA, the shHmga2 plus Hmga2 wt and the shHmga2 plus Hmga2 ATG wt transplants generate a highly metastatic disease, with lesions disseminating to both local and distant lymph nodes, kidney, and the abdominal and thoracic cavities (data not shown). We also examined the effect of Hmga2 ceRNA function on survival in the transplant system. We observed a dramatic reduction in survival in animals transplanted with the Hmga2 wt and ATG wt cells, similar to that seen with transplant of the control shRNA cells (Fig. 2d); we noted a more modest reduction in survival with transplant of the Hmga2 $\mathrm{m} 7$ cells. In total, these findings indicate that Hmga2 competing RNA activity dramatically promotes in vivo lung cancer formation.

To elucidate the mechanism of Hmga2 ceRNA function on lung carcinogenesis, we analysed the set of genes differentially expressed between metastatic and non-metastatic $\operatorname{Kras}^{G 12 D}$;Trp5 $3^{\text {floxfflox }}$ lung cancer cells ${ }^{6}$ and compared them to the list of predicted let-7 target genes based upon the miRNA target prediction algorithm TargetScan ${ }^{14}$ (Supplementary Table 5). Kras was not a candidate in this analysis, in spite of previous description of Kras as an important let-7 target ${ }^{15}$. Moreover, the Hmga2 allelic series had no impact on either expression of K-Ras protein or activity of downstream K-Ras signalling pathways (Extended Data Fig. 3c). In contrast, we observed several known Hmga2 transcriptional targets, including components of the Igf $2 \mathrm{bp} \mathrm{family}{ }^{16}$, validating this approach.

To elucidate more broadly which transcripts are Hmga 2 ceRNA targets, we initially examined whether let-7 sites are enriched among transcripts induced by the Hmga 2 ceRNA through RNA-seq of the 482N1 allelic series combined with Sylamer analysis, which detects miRNA seed sites as nucleotide strings enriched within the $3^{\prime}$ UTRs of transcripts ${ }^{17}$. We first compared control to Hmga2 knockdown cells and observed a specific enrichment of let-7 sites lost with Hmga2 depletion (Extended Data Fig. 4a). We then determined if this was specific to ceRNA activity by determining let-7 site enrichment upon re-expression of either the Hmga2 wt or ATG wt constructs in Hmga2 knockdown cells. In both conditions, let- 7 sites were enriched among the up-regulated transcripts (Extended Data Fig. 4b and c) Importantly, let-7 sites were not enriched with re-expression of either Hmga2 m7 or ATG $\mathrm{m} 7$ in the Hmga2-depleted background (Extended Data Fig. 4d and e). Moreover, analysis of fragments per kilobase of exon per million fragments mapped (FPKM) in the RNA-seq results from control 482N1 cells showed Hmga2 was among the most highly expressed predicted let-7 target transcripts, suggesting that Hmga2 constitutes a physiologically germane fraction of the let-7 target milieu (Supplementary Table 6). Taken together, these results indicate that the Hmga 2 ceRNA broadly regulates let-7 targets.

To more specifically assess Hmga2 ceRNA targets, we examined which transcripts were suppressed in response to Hmga2 depletion; 13 out of 34 predicted targets were suppressed 
by Hmga2 knockdown (Extended Data Fig. 5a). To delineate which of these were Hmga2 transcriptional targets versus ceRNA targets, we re-expressed either Hmga2 wt or ATG wt in knockdown cells. As seen in Extended Data Figure 5b, 6 out of 13 transcripts were rescued by both Hmga2 wt and ATG wt, suggesting they are putative ceRNA targets; the remaining targets were rescued only by Hmga2 wt, suggesting they are targets of Hmga2 transcription factor function. These Hmga2 ceRNA targets were strikingly enriched in let-7 regulated transcripts, as their repression by Hmga2 loss could be reversed with the use of a 'tough decoy' let-7 sponge transcript, designed to be an efficient and long-term suppressor of miRNA function (Extended Data Fig. $5 \mathrm{c})^{18}$. Conversely, over-expression of let-7 suppressed these transcripts, although Hmga2 transcriptional targets were also affected due to depletion of Hmga2 (Extended Data Fig. 5d). Taken together, these studies outline a collection of putative target transcripts regulated by Hmga2 ceRNA function.

Among these six Hmga 2 ceRNA target transcripts, we found the TGF- $\beta$ co-receptor $T g f b r 3^{12}$ to be both up-regulated in metastatic lung cancer cells and a putative let-7 target. Furthermore, several Hmga 2 ceRNA targets have been described as targets of TGF- $\beta$ signalling ${ }^{19}$. Thus, we examined whether Hmga2 exerts ceRNA function through enhanced TGF- $\beta$ signalling via Tgfbr3. In line with this, we found that in both $368 \mathrm{~T} 1$ and $482 \mathrm{~N} 1$ cells, Hmga2 wt and ATG wt promote the expression of Tgfbr3 protein (Fig. 3a). This Tgfbr3 upregulation also occurs to a lesser degree at the mRNA level, as has been described previously for miRNA $\operatorname{targets}^{20}$ (Fig. 3b). Moreover, exogenous expression of let-7 reversed the ability of the Hmga2 ceRNA to up-regulate Tgfbr3, suggesting this effect is controlled by let-7 (Extended Data Fig. 2a). An important consideration in ceRNA/target analysis is the absolute levels of $\mathrm{Hmga2}$, Tgfbr3, and let-7 transcripts in cells, so we determined the copies per cell of these factors (Extended Data Fig. 4f). We observed that Hmga2 and Tgfbr3 had similar levels of transcript, as one might expect for two factors that can titrate expression of one another; similar results were observed in FPKM analysis of these transcripts in control $482 \mathrm{~N} 1$ cells by RNA-seq (Supplementary Table 6). Furthermore, total let-7 family expression was within an order of magnitude of Hmga2 and Tgfbr3. As this pool of let-7 regulates the entire target set, one could expect miRNA occupancy to be a limiting factor, allowing for the contribution of a ceRNA-like Hmga2. Taken together, these results suggest that Hmga 2 could regulate Tgfbr 3 expression as a let-7 ceRNA.

In line with these observations of Hmga2 promoting Tgfbr3 expression, Hmga2 wt and ATG wt activated TGF- $\beta$ signalling via phosphorylation of Smad2 (Fig. 3a). This effect was let-7 dependent, as exogenous let-7 suppressed Smad2 phosphorylation (Extended Data Fig. 2a). That the TGF- $\beta$ pathway is active in the absence of exogenous ligand is likely due to low but detectable levels of TGF- $\beta$ in serum during cell culture ${ }^{21}$. We further examined whether Hmga 2 ceRNA function affects TGF- $\beta$ pathway activation by two methods. First, we found that a TGF- $\beta$ reporter (CAGA12) ${ }^{22}$ was potently induced by Hmga 2 wt and ATG wt (Extended Data Fig. 5e). Second, analysis of TGF- $\beta$ target transcript levels revealed specific expression of these genes with the Hmga2 wt and ATG wt constructs (Extended Data Fig. 5f). Notably, we observed little activation of the TGF- $\beta$ pathway by Hmga2 $\mathrm{m} 7$, in spite of previous reports of Hmga2 functioning as a Smad2/3/4 co-activator in the epithelialmesenchymal transition (EMT) ${ }^{23}$; this is likely due to the lack of upstream activation of the pathway. In line with this, the Hmga2 ceRNA does not induce an EMT in either 368T1 or 482N1 cells (Extended Data Fig. 6a). Overall, these results indicate that the Hmga2 ceRNA induces expression of Tgfbr3 and potentiates TGF- $\beta$ signalling.

To determine whether the effects of Hmga2 on Tgfbr3 occur via let-7 mediated derepression, we first examined the effect of $\mathrm{Hmga} 2$ ceRNA function on a reporter containing the Tgfbr $33^{\prime}$ UTR. In both $368 \mathrm{~T} 1$ and 482N1 cells, Hmga2 wt and ATG wt induced expression of luciferase under the control of the wild type Tgfbr $33^{\prime}$ UTR, but not if the let-7 
site was mutated (Fig. 3c). Furthermore, we found that the effect of the Hmga2 ceRNA was broadly miRNA dependent, as Hmga2 wt and ATG wt induced the reporter expression in Dicerl-intact sarcoma cells, but not in a Dicerl-null derivative cell line ${ }^{24}$ (Extended Data Fig. 6b). To assess directly whether Hmga2 induces $T g f b r 3$ via competition away from Ago2, we performed RNA immunoprecipitation (RIP) on Ago2 in lung cancer cells expressing the Hmga2 allelic series. As seen in Figure 3d, we found that Hmga2 wt and ATG wt were as comparably recruited to Ago2 as endogenous Hmga2 while Hmga2 m7 and ATG $\mathrm{m} 7$ were not. Moreover, Hmga2 wt and ATG wt cells had a substantial decrease in $T g f b r 3$ recruitment to Ago2. Of note, these effects on Ago2 occupancy by Tgfbr3 are not caused by a change in let-7 activity, as various let-7 family members were comparably loaded on Ago2 across the Hmga2 allelic series (Extended Data Fig. 7). Thus, these results demonstrate Hmga2, via its let-7 binding sites, displaces Tgfbr3 from miRNA-mediated repression by RISC. In total, these results suggest the Hmga 2 ceRNA directly functions by blocking recruitment of $T g f b r 3$ to the Ago2-based miRNA repression complex.

To examine whether Hmga2 ceRNA activity through Tgfbr3 is functionally relevant, we used shRNAs to deplete $T g f b r 3$ in $482 \mathrm{~N} 1$ cells and 368T1 cells expressing either Hmga2 wt or ATG wt. At both the mRNA and protein level, multiple shRNAs reduced Tgfbr3 expression (Figs. 3e and f). Moreover, knockdown of Tgfbr3 led to substantial suppression of TGF- $\beta$ signalling, as evinced by loss of Smad 2 phosphorylation, CAGA12 reporter activity and expression of TGF- $\beta$ target genes (Fig. 3f and Extended Data Fig. 8a and b). We then assessed the functional effect of Tgfbr3 loss on Hmga2 ceRNA-driven soft agar colony formation. In all cells, $T g f b r 3$ knockdown strikingly suppressed anchorage-independent growth, though not to the same extent as Hmga2 depletion in 482N1 cells (Fig. 3g). This occurred without generally affecting proliferation, as measured by BrdU incorporation (Extended Data Fig. 8c). We further functionally analysed the broader set of six Hmga2 ceRNA targets by individually depleting them by siRNA (Extended Data Fig. 8d). When we compared their effects on anchorage-independent growth, both Hmga2 and Tgfbr3 loss strongly suppressed growth, Hmgal depletion modestly reduced colony formation, while the remaining targets had little effect (Extended Data Fig. 8e). It should be noted that these other targets include extracellular factors like Angptl2 and Col1a2 that might promote lung cancer progression in vivo in a non-cell autonomous manner, and could thus still be relevant to Hmga 2 ceRNA activity in lung cancer progression. This considered, these results still indicate that $T g f b r 3$, though certainly not the only relevant Hmga2 ceRNA target, is an important effector of Hmga2 ceRNA function in lung cancer cell transformation.

To determine if this effect of Tgfbr3 is driven through potentiation of TGF- $\beta$ signalling, we inhibited the TGF- $\beta$ pathway with the TGF- $\beta$ receptor kinase inhibitor SB-431542 (SB) ${ }^{25}$. In 368T1 and 482N1 cells, SB treatment led to a substantial inhibition of Smad2 phosphorylation (Extended Data Fig. 9a). In addition, SB treatment of 482N1 and 368T1 Hmga2 wt and ATG wt cells markedly suppressed CAGA12 reporter activity and expression of TGF- $\beta$ target genes (Extended Data Fig. $9 b$ and c). We then examined whether SB could inhibit Hmga 2 ceRNA driven soft agar colony formation and observed a striking reduction in anchorage-independent growth (Extended Data Fig. 9d). This impaired colony formation was not due to general proliferative arrest, as SB treated cells had a similar rate of BrdU incorporation (Extended Data Fig. 9e). Strikingly, many Hmga2 ceRNA targets are in fact TGF- $\beta$ target genes, as their expression is suppressed by SB treatment and induced by exogenous addition of TGF- $\beta$ (Extended Data Fig. 9f and g). Thus, it is possible that Hmga2 could function in a feed forward loop in which it regulates TGF- $\beta$ target genes directly through ceRNA function and indirectly through TGF- $\beta$ signalling via Tgfbr3. In sum, these results indicate that TGF- $\beta$ signalling via Tgfbr 3 is an important pathway downstream of Hmga 2 ceRNA function. 
Based upon the above findings, we wanted to explore if $H M G A 2$ functions as a ceRNA for $T G F B R 3$ (the human orthologues of Hmga2 and $T g f b r 3$, respectively) in NSCLC patients. An important corollary of the ceRNA hypothesis is the coordinate regulation of a competing RNA and its targets, such that up-regulation of the ceRNA should lead to higher expression of the target RNA and vice versa ${ }^{10}$. To assess this, we used NSCLC gene expression data generated by the Cancer Genome Atlas (TCGA) and sorted the patient cohort into the top and bottom quartiles of HMGA2 expression (HMGA2 high and low, respectively) (Fig. 4a). As seen in Figure 4b, we observed significantly higher levels of TGFBR3 transcript in $H M G A 2$ high versus low patient samples. To address the converse relationship, we sorted the TCGA data set into top and bottom quartiles of TGFBR3 expression (TGFBR3 high and low, respectively) (Fig. 4c). When we compared $H M G A 2$ transcript levels between the groups, we found $H M G A 2$ to be significantly over-expressed in $T G F B R 3$ high versus low patient samples (Fig. 4d). To extend and validate these findings, we performed similar gene expression analyses of HMGA2 and TGFBR3 in an independent lung adenocarcinoma patient gene expression cohort, the Director's Challenge data $\operatorname{set}^{26}$. Similar to the findings with the TCGA cohort, we observed HMGA2 and TGFBR3 to be co-ordinately expressed in the Director's Challenge data set (Extended Data Fig. 10a-d). While we focused specifically on high and low expressors of $H M G A 2$ and $T G F B R 3$, where ceRNA activity is more likely to occur ${ }^{10}, H M G A 2$ and $T G B R 3$ expression was broadly correlated across both data sets (Extended Data Fig. 10e and f). As these two data sets constitute two of the largest collections of NSCLC gene expression data available, we believe these findings are in line with $H M G A 2$ functioning as a ceRNA for TGFBR3 in NSCLC patients. It is possible that the co-expression of $H M G A 2$ and TGFBR3 could correspond to additional tumour characteristics these data sets do not include; future studies in independent data sets would be needed to assess this issue. In total, our results suggest a model by which Hmga 2 promotes lung cancer progression by competing for let-7 occupancy with other targets, including $T g f b r 3$, leading to the up-regulation of those targets (Fig. 4e). Importantly, this occurs without changes in the levels of let-7 family microRNAs, reflecting specific competition for microRNA binding among targets.

Here we have outlined a novel gene expression pathway in which a protein-coding gene, Hmga2, operates largely independently of its protein-coding function to promote lung cancer progression as a competing endogenous RNA. While much of this ceRNA activity is driven by over-expression of TGF- $\beta$ signalling via Tgfbr3, there are likely additional Hmga 2 ceRNA targets to be found in future studies. Moreover, $H M G A 2$ is over-expressed in many other cancer types ${ }^{27}$, making it possible that $H M G A 2$ functions as a ceRNA in cancer sites beyond lung. Taken more broadly, these findings raise the possibility that many proteincoding genes differentially expressed in cancer might contribute to tumourigenesis through this distinct mode of regulatory gene expression. Moreover, these results raise issues with the validation of candidates in RNA interference screens. The "gold standard" assay for validating an siRNA target is expression of an siRNA-resistant form of the coding sequence $^{28}$; however, such an approach overlooks the possibility that depletion of both the full-length RNA and protein might contribute to a given phenotype, requiring complementation by the full-length transcript. Such dual-function ceRNA/protein activities necessitate a deeper exploration of the coding genome in biological systems.

\section{Online-only Methods}

\section{Cell lines}

$368 \mathrm{~T} 1$ and $482 \mathrm{~N} 1$ cells were generated previously ${ }^{6}$. KPD F/- and -/- sarcoma cells were a kind gift of Phillip A. Sharp ${ }^{24}$. Cancer Research UK Cell Services provided HEK293, Phoenix ampho, NCI-H1299, and SK-MES-1 cells. All cell lines were cultured in DMEM 
medium with $10 \% \mathrm{FBS}, 10 \mathrm{mM}$ glutamine and $1 \%$ penicillin and streptomycin at $37^{\circ} \mathrm{C}$ in a $5 \% \mathrm{CO}_{2} / 95 \%$ air incubator.

\section{Drug and reagent treatment}

For Western, luciferase, qRT-PCR, and BrdU analysis, 368T1 cells were co-treated with 2 ng/mL TGF- $\beta$ and either DMSO or $2.5 \mu \mathrm{M}$ SB-431542 ("SB") and 482N1 cells were treated with either DMSO or $2.5 \mu \mathrm{M}$ SB-431542 for 24 hours. For soft agar analysis, cells were fed with media containing either DMSO or SB.

For Western analyses, 368T1 and $482 \mathrm{~N} 1$ cells were plated $\left(2.5 \times 10^{5}\right.$ cells $)$ into 6-well plates overnight. Cells were then treated with DMSO, $1 \mu \mathrm{M}$ AZD6244 or $1 \mu \mathrm{M}$ PIK-75 for two hours and lysates were prepared.

\section{Lentivirus and retrovirus production}

Short hairpin RNA (shRNA) lentiviruses (which are listed in Supplementary Table 1) were generated by co-transfection of HEK293 cells with shRNA vector and packaging vectors pCMV-VSVG and pCMV-8.2. MSCV-based retroviruses were generated by transfection of Phoenix ampho cells with retroviral vectors. 48 and 72 hours after transfection, virus particles in the medium were harvested, filtered, and transduced onto cells.

\section{Stable cell line generation}

Expression vectors were initially linearized overnight using BglII. Linearised DNA was then transfected into cells in 24-well plates, with media replaced six hours later. Cells were then replated into 6-well plates in the presence of $500 \mu \mathrm{g} / \mathrm{mL}$ G418 for selection over two weeks. For lentiviral and retroviral infection, cells were infected in 6-well plates and subsequently split into $10 \mathrm{~cm}$ dishes in the presence of $2.5 \mu \mathrm{g} / \mathrm{mL}$ puromycin for selection over 72 hours.

\section{Western blotting}

Western blotting was performed by standard methods. Antibody details are provided in Supplementary Table 2.

\section{qRT-PCR}

RNA was isolated using RNA-Bee using manufacturers' instructions. Both standard and miRNA-specific qRT-PCR was performed using QuantiTect SYBR Green primers as per manufacturers' instructions (QIAGEN). Primer details are provided in Supplementary Table 3.

\section{Luciferase assays}

CAGA12-Cells were seeded into 96-well plates in triplicate and co-transfected with pRLTK (Promega) and either control pGL3 or the TGF- $\beta$ reporter pCAGA12-GL3 ${ }^{22} .24$ hours later, luciferase activities were determined using the Dual-Luciferase Assay System as per manufacturers' instructions (Promega).

Tgfbr3 3' UTR-Cells were seeded into 96-well plates in triplicate and co-transfected with pGL3 (Promega) and either the control pRL-CXCR $4^{30}$ or the wild type/mutant fragment of the $T g f b r 33^{\prime}$ UTR. Luciferase activities were determined as above.

\section{BrdU analysis}

Cells were plated in triplicate into six-well plates overnight $\left(2.5 \times 10^{5}\right.$ cells per well). They were then treated with $10 \mu \mathrm{M}$ BrdU for one hour, trypsinized, washed in PBS and stained 
for BrdU/DAPI analysis using standard methods by the Cancer Research UK Flow Cytometry Facility.

\section{SiRNA/miRNA transfection}

Cells were transfected with siRNA/miRNA as described previously ${ }^{29}$. Cells were subsequently plated into soft agar assays as above or harvested for RNA/protein as above.

\section{polyHEMA plating analysis}

6-well plates were either mock coated or coated with (polyHEMA) as previously described ${ }^{31}$. Cells were subsequently plated into these 6-wells overnight and BrdU analysis was performed as described above.

\section{Histopathological analysis}

After sacrifice by $\mathrm{CO}_{2}$ asphyxiation, lungs were collected from animals and fixed overnight in $10 \%$ formalin. They were subsequently placed in $70 \%$ ethanol and submitted for embedding, sectioning, and hematoxylin and eosin (H\&E) staining.

\section{In vitro transcription}

TOPO cloned fragments of Hmga2 and Tgfbr3 were in vitro transcribed using the SP6 MAXIscript kit as per manufacturers' instructions (Ambion). RNA was subsequently purified using the RNEasy cleanup kit as per manufacturers' instructios (Qiagen).

\section{Transcript copy number analysis}

For determination of transcript copy number, RNA was prepared from $2 \times 10^{6} 482 \mathrm{~N} 1$ cells using RNA Bee as described above. qRT-PCR was performed for Hmga2, Tgfbr3, and let-7a/c/e/f/i as described above. $\mathrm{C}_{\mathrm{T}}$ values were compared to a two-fold dilution series of either in vitro transcribed Hmga2/Tgfbr3 or synthetic let-7a/c/e/f/i miRNA mimics (Qiagen). Moles of transcript and copies of transcript per cell were then calculated using standard stoichiometric methods. Confidence intervals were calculated using a t-statistic.

\section{RNA library production and next-generation sequencing (RNA-seq)}

Total RNA was generated from the 482N1 Hmga2 allelic series (with biological triplicates) as described above. The Total RNA samples were initially quality controlled (QC) using the 6000 Nano RNA Chip on the BioAnalyser 2100 (Agilent, Santa Clara, CA, USA) to ensure RNA integrity and estimate concentration before starting the procedure. If the samples passed the initial QC the Total RNA samples were subjected to poly-A selection using SeraMag oligo (dT) beads (Thermo Fisher Scientific Inc.), and the bound poly-A RNA species utilized for downstream library prep using the Illumina mRNA kit RS-122-2101 (TruSeq Stranded mRNA Sample Prep. Illumina San Diego, CA, USA). The standard PCR cycles suggested in the protocol were also altered to match the concentration of the total RNA from the initial QC. After production of the mRNA libraries a final QC was performed on a DNA 1000 chip using the BioAnalyser 2100 (Agilent, Santa Clara, CA, USA). If the mRNA libraries passed the QC they were ready for flow cell cluster formation on a cBot and then 100 b.p. Paired End (PE) sequencing by synthesis on the HiSeq 2500 was performed.

\section{RNA-seq data processing and analyses}

We aligned 100 b.p. paired-end sequencing reads to the mouse genome ( $\mathrm{mm} 9, \mathrm{UCSC})$ using Tophat $2 /$ Bowtie $2^{32}$ allowing for 5 mismatches, a mate-inner-distribution of -40 and a matestandard-deviation of 50. These library fragment metrics were derived from an alignment of $5 \mathrm{e} 5$ sampled reads to the mouse transcriptome. We identified read-pair mappings to gene 
structures derived from RefSeq (RefGene table, UCSC) using the summarizeOverlaps function with mode IntersectStrict (GenomicRanges, Bioconductor). This generated a mean fragments per sample value of 33.5e6. Using these raw counts we identified genes expressed across the sample groups. We removed genes with a count $<=10$ across all samples prior to statistical analysis. The differential analysis was carried out using edge $\mathrm{R}^{33}$ applying TMM library normalisation and a 0.0005 false discovery rate (fdr) to select expressed transcripts. In addition, we calculated gene level fragments per kilobase of exon per million fragments mapped (FPKM) values for the same gene set using Cufflinks ${ }^{34}$.

\section{Sylamer analysis}

A list of Ensembl gene IDs was generated from the set of transcripts expressed in the samples described above. This set of transcripts was then placed in rank order by their differential expression between two groups and analysed using the Sylamer algorithm via the SylArray platform ${ }^{17}$. A Sylamer plot was then generated for all 8 mer miRNA strings whose target sites were enriched significantly ( $<<0.001$ by hypergeometric testing).

\section{Statistical testing}

Unless otherwise specified, statistical significance was assessed by the Student's t-test.

\section{Vector cloning}

Hmga2-pCDNA3.1-Hmga2 wt and Hmga2 m7 ("wt" and "m7") were acquired from Addgene (Plasmid nos. 14789 and 14792) ${ }^{13}$. In order to generate pCDNA3.1-Hmga2 ATG wt and pCDNA3.1-Hmga2 ATG m7 ("ATG wt" and "ATG m7"), the wt and $m$ 7 constructs were mutated by site-directed mutagenesis as per manufacturers' instructions (Stratagene) using primers 5'-GGTAGCGGCGGCGGGAGGCAGGCTGAGCGCACGCGGTGAGG-3' and 5'-CCTCACCGCGTGCGCTCAGCCTGCCTCCCGCCGCCGCTACC-3'. For generation of 482N1 shHmga2 stable cell lines, "wt", "m7", "ATG wt" and "ATG m7" the shRNA binding site was mutated using primers $5^{\prime}$ -

CCATTTCTGCAAGCTAAGTATGTTTGCAGGAGCCCCGGCTCCGGACGCGTAACT G CATCCAACTTTCTCC-3' and 5'GGAGAAAGTTGGATGCAGTTACGCGTCCGGAGCCGGGGCTCCTGCAAACATACT TAGCTTGCAGAAATGG-3'.

pRL-Tgfbr3-A fragment containing the Tgfbr3 3'UTR was PCR amplified from 482N1 cDNA using primers 5'-TAAGAACTCGAGCTGCGTGTGTTCTCCGCAG-3' and 5'TAACAAGCGGCCGCCTGTCAGTTTAATGAACGAACC- $3^{\prime}$. Both the PCR product and pRL-CXCR4 were digested with XhoI and NotI and ligated together to generate pRLTgfbr3. The let- 7 mutant was generated by site-directed mutagenesis as above using primers $5^{\prime}-$

GCAGGCGCGTGCCTGTCTTTTTGTACTGTAACGGGCTCATGGTTTGAATGATGAG CGTACTGCTGGTTG-3' and 5'-

CAACCAGCAGTACGCTCATCATTCAAACCATGAGCCCGTTACAGTACAAAAAGA CAGGCACGCGCCTGC-3'.

Hmga2-TOPO/Tgfbr3-TOPO-A fragment containing the Hmga2 cDNA sequence recognized by the respective qRT-PCR primers was PCR amplified from 482N1 cDNA using primers 5'-CTACATCCCGTCTCCCGAAAGGTGCTGG-3' and 5'GGATCCGGTAGAAATTGAATGTCGGCGCCCCCTAATC- $3^{\prime}$. A fragment containing the Tgfbr 3 cDNA sequence recognized by the respective qRT-PCR primers was PCR amplified from 482N1 cDNA using primers $5^{\prime}$ CTCGAGCAGAAGAAGTGCAAGGGGGCGTGAATATCG-3' and 5'- 
CCATGTTGAAGGTAGCATTTCCATCGAGCTGGTCCTGGAAG-3' ${ }^{\prime}$. PCR products were subsequently TOPO cloned as per manufacturers' instructions (Invitrogen) and used as substrates for in vitro transcription.

Hmga2 wt/m7 3'UTR_A fragment containing the Hmga2 3'UTR was PCR amplified from the Hmga2 wt or Hmga2 m7 pcDNA3.1 constructs used for transfection. Products were amplified using primers $5^{\prime}$-GCAGAATTCGGGGCGCCGACATTCAATTTC- $3^{\prime}$ and 5'-TAAGCGGCCGCGCCCACAGAGGCTGTTATGTTTATTG-3'. PCR products were subsequently TOPO cloned as per manufacturers' instructions (Invitrogen). TOPO clone inserts and pcDNA3.1-Hmga2 wt were EcoRI digested and ligated together to generate pcDNA3.1-Hmga2 wt 3'UTR and pcDNA3.1-Hmga2 m7 3'UTR.

\section{Supplementary Material}

Refer to Web version on PubMed Central for supplementary material.

\section{Acknowledgments}

We thank the laboratory of T. Jacks for providing the 482N1 and 368T1 lung cancer cell lines and the laboratories of P.A. Sharp and P. Chambon for providing the KPD sarcoma cell lines. We thank the LRI FACS Facility for the BrdU analyses and the LRI Biological Resources Unit for assistance with the animal studies. We thank M.S. Ebert and E. De Bruin for critical review of the manuscript. M.S.K. is a Long-Term Fellow of the Human Frontier Science Program and the European Molecular Biology Organization. This work was funded by Cancer Research UK and by the European Commission's Seventh Framework Programme (FP7/2007-2013) under the grant agreement Lungtarget (project $\mathrm{n}^{\circ} 259770$ ).

\section{References}

1. Ferlay J, et al. Estimates of worldwide burden of cancer in 2008: GLOBOCAN 2008. Int J Cancer. 2010; 127:2893-2917. [PubMed: 21351269]

2. Herbst RS, Heymach JV, Lippman SM. Lung cancer. N Engl J Med. 2008; 359:1367-1380. [PubMed: 18815398]

3. Di Cello F, et al. HMGA2 participates in transformation in human lung cancer. Mol Cancer Res. 2008; 6:743-750. [PubMed: 18505920]

4. Meyer B, et al. HMGA2 overexpression in non-small cell lung cancer. Mol Carcinog. 2007; 46:503511. [PubMed: 17477356]

5. Sarhadi VK, et al. Increased expression of high mobility group A proteins in lung cancer. J Pathol. 2006; 209:206-212. [PubMed: 16521118]

6. Winslow MM, et al. Suppression of lung adenocarcinoma progression by Nkx2-1. Nature. 2011; 473:101-104. [PubMed: 21471965]

7. Ebert MS, Neilson JR, Sharp PA. MicroRNA sponges: competitive inhibitors of small RNAs in mammalian cells. Nat Methods. 2007; 4:721-726. [PubMed: 17694064]

8. Karreth FA, et al. In vivo identification of tumor-suppressive PTEN ceRNAs in an oncogenic BRAF-induced mouse model of melanoma. Cell. 2011; 147:382-395. [PubMed: 22000016]

9. Poliseno L, et al. A coding-independent function of gene and pseudogene mRNAs regulates tumour biology. Nature. 2010; 465:1033-1038. [PubMed: 20577206]

10. Salmena L, Poliseno L, Tay Y, Kats L, Pandolfi PP. A ceRNA hypothesis: the Rosetta Stone of a hidden RNA language? Cell. 2011; 146:353-358. [PubMed: 21802130]

11. Tay Y, et al. Coding-independent regulation of the tumor suppressor PTEN by competing endogenous mRNAs. Cell. 2011; 147:344-357. [PubMed: 22000013]

12. Wang XF, et al. Expression cloning and characterization of the TGF-beta type III receptor. Cell. 1991; 67:797-805. [PubMed: 1657407]

13. Mayr C, Hemann MT, Bartel DP. Disrupting the pairing between let-7 and Hmga2 enhances oncogenic transformation. Science. 2007; 315:1576-1579. [PubMed: 17322030] 
14. Friedman RC, Farh KK, Burge CB, Bartel DP. Most mammalian mRNAs are conserved targets of microRNAs. Genome Res. 2009; 19:92-105. [PubMed: 18955434]

15. Johnson SM, et al. RAS is regulated by the let-7 microRNA family. Cell. 2005; 120:635-647. [PubMed: 15766527]

16. Brants JR, et al. Differential regulation of the insulin-like growth factor II mRNA-binding protein genes by architectural transcription factor HMGA2. FEBS Lett. 2004; 569:277-283. [PubMed: 15225648]

17. van Dongen S, Abreu-Goodger C, Enright AJ. Detecting microRNA binding and siRNA off-target effects from expression data. Nature methods. 2008; 5:1023-1025. [PubMed: 18978784]

18. Xie J, et al. Long-term, efficient inhibition of microRNA function in mice using rAAV vectors. Nat Methods. 2012; 9:403-409. [PubMed: 22388288]

19. Massague J. TGFbeta signalling in context. Nat Rev Mol Cell Biol. 2012; 13:616-630. [PubMed: 22992590]

20. Guo H, Ingolia NT, Weissman JS, Bartel DP. Mammalian microRNAs predominantly act to decrease target mRNA levels. Nature. 2010; 466:835-840. [PubMed: 20703300]

21. Oida T, Weiner HL. Depletion of TGF-beta from fetal bovine serum. J Immunol Methods. 2010; 362:195-198. [PubMed: 20837018]

22. Dennler S, et al. Direct binding of Smad3 and Smad4 to critical TGF beta-inducible elements in the promoter of human plasminogen activator inhibitor-type 1 gene. EMBO J. 1998; 17:3091-3100. [PubMed: 9606191]

23. Thuault S, et al. HMGA2 and Smads co-regulate SNAIL1 expression during induction of epithelial-to-mesenchymal transition. J Biol Chem. 2008; 283:33437-33446. [PubMed: 18832382]

24. Ravi A, et al. Proliferation and tumorigenesis of a murine sarcoma cell line in the absence of DICER1. Cancer Cell. 2012; 21:848-855. [PubMed: 22698408]

25. Inman GJ, et al. SB-431542 is a potent and specific inhibitor of transforming growth factor-beta superfamily type I activin receptor-like kinase (ALK) receptors ALK4, ALK5, and ALK7. Mol Pharmacol. 2002; 62:65-74. [PubMed: 12065756]

26. Shedden K, et al. Gene expression-based survival prediction in lung adenocarcinoma: a multi-site, blinded validation study. Nat Med. 2008; 14:822-827. [PubMed: 18641660]

27. Park SM, et al. Let-7 prevents early cancer progression by suppressing expression of the embryonic gene HMGA2. Cell Cycle. 2007; 6:2585-2590. [PubMed: 17957144]

28. Kaelin WG Jr. Molecular biology. Use and abuse of RNAi to study mammalian gene function. Science. 2012; 337:421-422. [PubMed: 22837515]

29. Kumar MS, et al. The GATA2 transcriptional network is requisite for RAS oncogene-driven nonsmall cell lung cancer. Cell. 2012; 149:642-655. [PubMed: 22541434]

30. Doench JG, Petersen CP, Sharp PA. siRNAs can function as miRNAs. Genes Dev. 2003; 17:438442. [PubMed: 12600936]

31. Liau SS, Jazag A, Ito K, Whang EE. Overexpression of HMGA1 promotes anoikis resistance and constitutive Akt activation in pancreatic adenocarcinoma cells. British journal of cancer. 2007; 96:993-1000. doi:10.1038/sj.bjc.6603654. [PubMed: 17342093]

32. Kim D, et al. TopHat2: accurate alignment of transcriptomes in the presence of insertions, deletions and gene fusions. Genome biology. 2013; 14:R36. doi:10.1186/gb-2013-14-4-r36. [PubMed: 23618408]

33. Robinson MD, McCarthy DJ, Smyth GK. edgeR: a Bioconductor package for differential expression analysis of digital gene expression data. Bioinformatics. 2010; 26:139-140. doi: 10.1093/bioinformatics/btp616. [PubMed: 19910308]

34. Trapnell C, et al. Differential analysis of gene regulation at transcript resolution with RNA-seq. Nature biotechnology. 2013; 31:46-53. doi:10.1038/nbt.2450. 

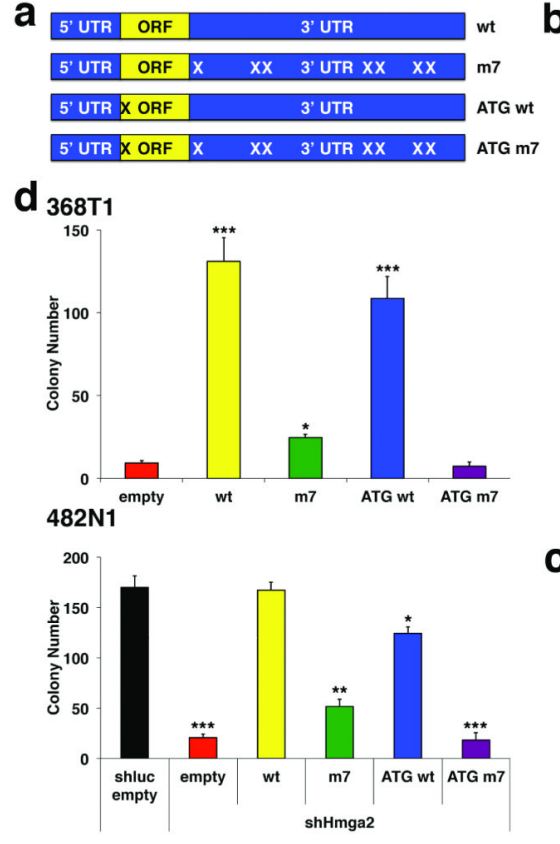

e $368 \mathrm{~T} 1$

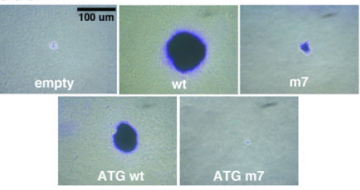

482N1

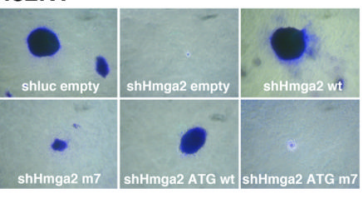

\section{b $368 \mathrm{T1}$}

non-metastatic, low Hmga2

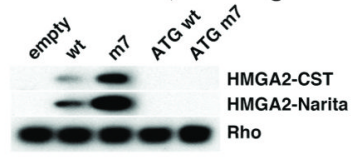

482N1

metastatic, high Hmga2

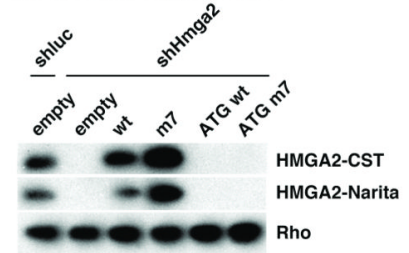

C $368 \mathrm{~T} 1$

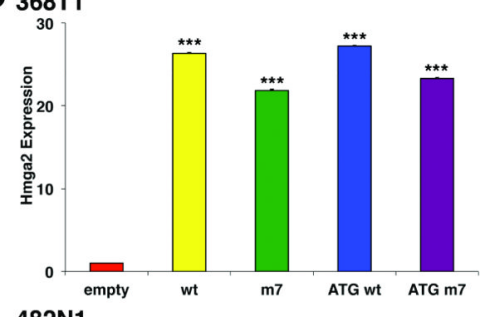

482N1

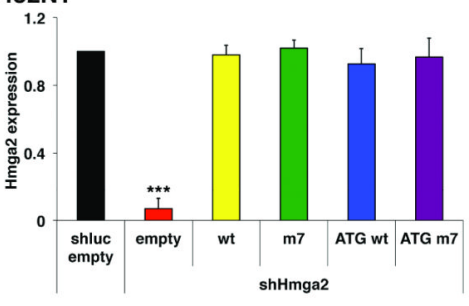

Figure 1. Hmga2 promotes lung cancer cell transformation in a protein-coding independent but let-7 site dependent manner

a, Diagram of Hmga2 allelic series: expression constructs containing the entire Hmga2 cDNA ("wt"); the cDNA with all seven let-7 sites in the $3^{\prime}$ UTR mutated ("m7"); the cDNA with the start codon mutated ("ATG wt"); and the cDNA with both the start codon and let-7 sites mutated ("ATG m7"). b, Hmga2 wt and $\mathrm{m} 7$ induce Hmga2 expression in non-

metastatic lung cancer cells (368T1) and restores expression cells in metastatic lung cancer cells (482N1) depleted for endogenous Hmga2 (shHmga2). Two distinct HMGA2 antibodies are used: one recognizes the N-terminus of HMGA2 (HMGA2-CST) and the other recognizes the central AT-hook region of HMGA2 (HMGA2-Narita). c, Hmga2 RNA is comparably expressed by the wt, m7, ATG wt, and ATG m7 in both 368T1 and 482N1 cells. Hmga 2 expression is normalized to Gapdh. 368T1 values are normalized to empty and $482 \mathrm{~N} 1$ values are normalized to shluc empty. Values are technical triplicates, have been performed independently three times, and represent mean $+/-$ standard deviation (s.d.) with propagated error. d, Hmga2 wt and ATG wt promote substantial anchorage-independent growth in both $368 \mathrm{~T} 1$ and $482 \mathrm{~N} 1$ cells. Values are technical triplicates, have been performed independently three times, and represent mean $+/-$ s.d. e, Representative images of soft agar colonies. Magnification is 10X. ***: $p<0.0005 ; * *$ : $p<0.005 ; *$ : $<<0.05$. 


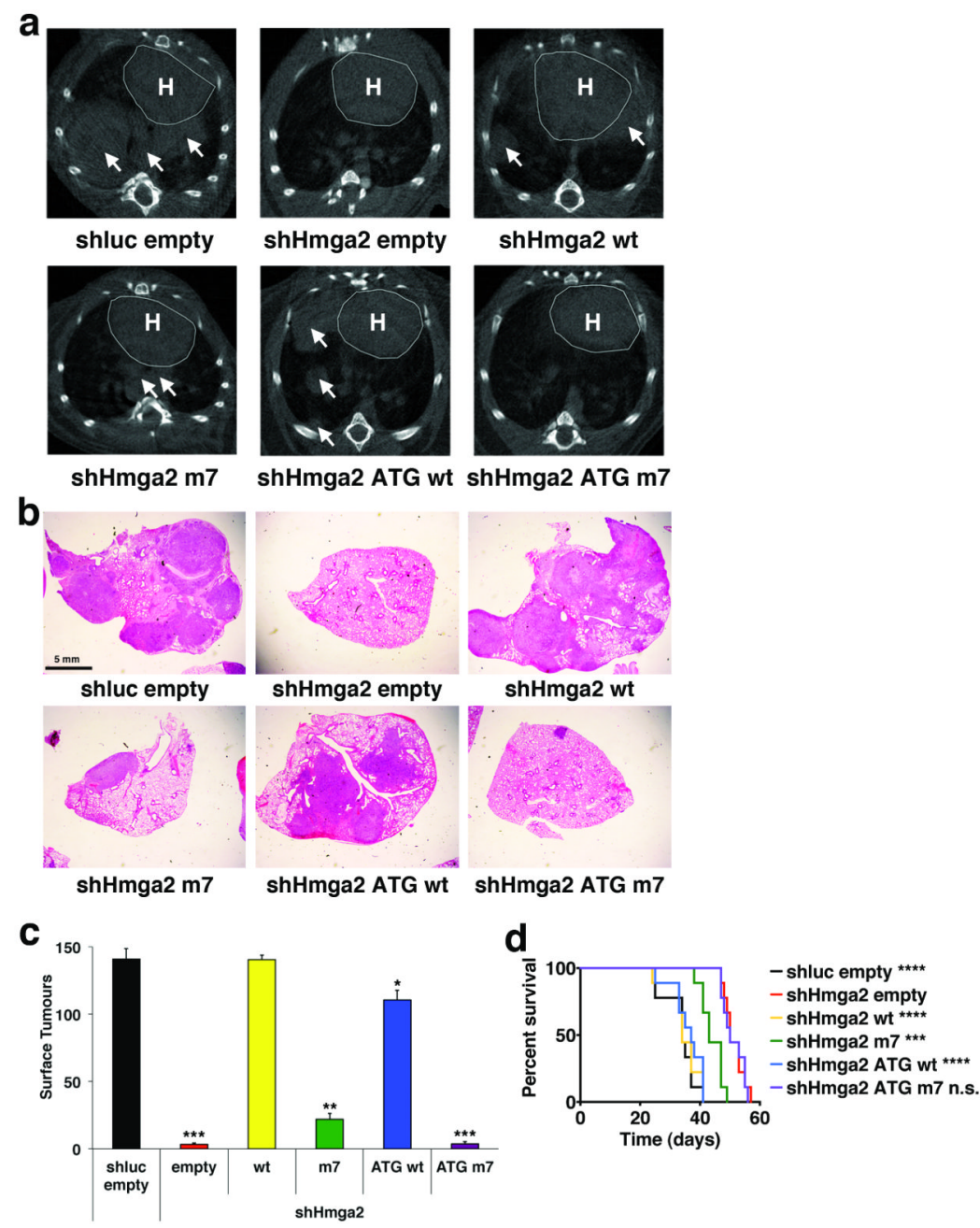

Figure 2. Hmga 2 ceRNA activity enhances lung cancer progression in vivo

a, Hmga2 wt and ATG wt restore lung tumour growth in response to endogenous Hmga2 knockdown. B6129SF1/Tac males were intravenously injected with 482N1 cells expressing either a control shRNA and empty vector (shluc empty) or shHmga2 with the Hmga2 allelic series. Three weeks afterwards, animals were scanned by micro-CT and representative transverse images are shown. The heart is demarcated (labelled ' $\mathrm{H}$ ') and white arrows identify lung tumours. b, Representative histological images of lungs transplanted with 482N1 cells from the series described in a. Magnification is 1X. c, Lung surface tumour counts were taken from animals transplanted with $482 \mathrm{~N} 1$ cells from the series described in a ( $\mathrm{n}=3$ animals per group). Values are technical triplicates represent mean $+/-$ s.e.m. d, Hmga 2 wt and ATG wt substantially reduce survival of animals transplanted with $482 \mathrm{~N} 1$ cells expressing the shRNA targeting Hmga2. Animals were intravenously transplanted with cells as in a. Animals were subsequently aged for survival and a Kaplan-Meier analysis was performed ( $\mathrm{n}=9$ animals per group). Median survival was 34 days for shluc empty/shHmga2 wt transplants; 37 days for shHmga2 ATG wt transplants; 43 days for shHmga2 m7 transplants; and 50 days for shHmga2 empty/ATG $\mathrm{m} 7$ transplants. Statistical significance was assessed by log-rank tests compared to shHmga2 empty. ****: p<0.00005; ***: $\mathrm{p}<0.0005 ; * *: \mathrm{p}<0.005 ; *: \mathrm{p}<0.05 ;$ n.s.: not significant. 


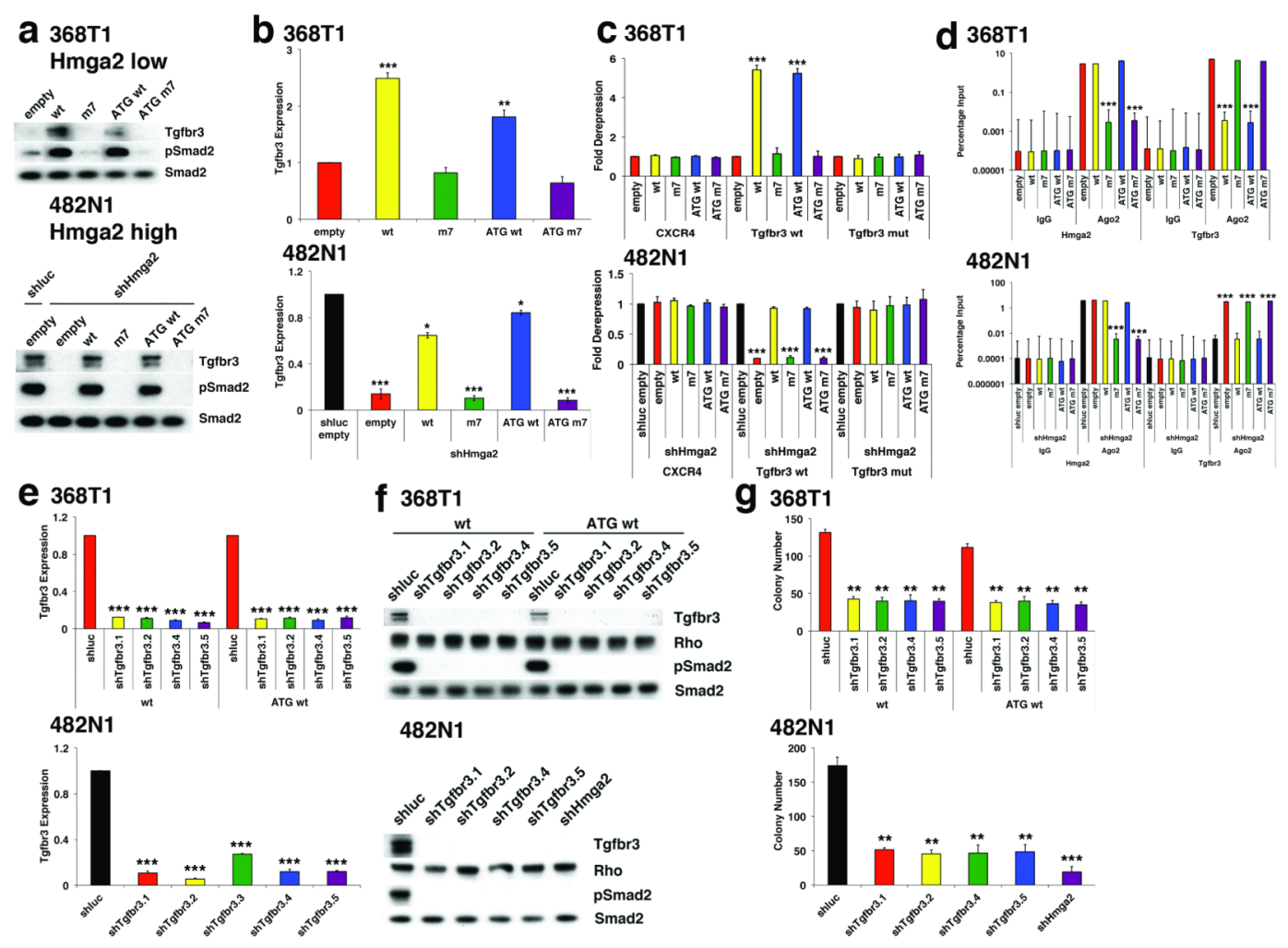

Figure 3. Hmga 2 ceRNA activity enhances TGF- $\beta$ signalling through over-expression of Tgfbr3 a, Hmga 2 wt and ATG wt substantially induce both Tgfbr3 protein expression and phosphorylation of Smad2 (pSmad2) in both 368T1 and 482N1 cells. b, Hmga2 wt and ATG wt significantly promote expression of Tgfbr3 mRNA in both 368T1 and 482N1 cells. $T g f b r 3$ expression is normalized to Gapdh. 368T1 values are normalized to empty and $482 \mathrm{~N} 1$ values are normalized to shluc empty. Values are technical triplicates, have been performed independently three times, and represent mean $+/-$ s.d. with propagated error. c, Hmga2 wt and ATG wt specifically induce expression of a luciferase Tgfbr3 $3^{\prime}$ UTR reporter in a let-7 site-dependent manner in both $368 \mathrm{~T} 1$ and $482 \mathrm{~N} 1$ cells. Cells were transfected with Renilla constructs of the control siCXCR4 multimer ${ }^{30}$ and either the Tgfbr3 wild type or let-7 mutant $3^{\prime}$ UTR reporter. Values are normalized to co-transfected pGL3 plasmid. 368T1 values are normalized to empty and 482N1 values are normalized to shluc empty. Values are technical triplicates, have been performed independently three times, and represent mean +/- s.d. with propagated error. d, Hmga2 wt and ATG wt displace Tgfbr3 from Argonaute-2 (Ago2) based RNA-induced silencing complexes. Lysates from 368T1 and 482N1 cells of the Hmga2 allelic series underwent either control immunoprecipitation (IgG) or immunoprecipitation for Ago2. RNA was purified and qRT-PCR was performed for Hmga 2 and Tgfbr3 on both the immunoprecipitated and input RNAs. Values are depicted as the percentage of input RNA, are technical triplicates, have been performed independently twice, and represent mean $+/-$ s.d. e, multiple shRNAs elicit substantial knockdown of Tgfbr3 mRNA in both $368 \mathrm{~T} 1$ and $482 \mathrm{~N} 1$ cells. $482 \mathrm{~N} 1$ cells were infected with control shRNA (shluc) or a set of shRNAs targeting Tgfbr3 (shTgfbr3.1-3.5), while 368T1 wt and ATG wt cells were infected with shluc or shTgfbr3.1, 3.2, 3.4, and 3.5. RNA was purified and qRT-PCR was performed. Tgfbr3 expression is normalized to Gapdh and 368T1 wt and ATG wt and 482N1 values are normalized to shluc. Values are technical triplicates, have been performed independently three times, and represent mean $+/-$ s.d. with 
propagated error. $\mathbf{f}$, multiple shRNAs induce knockdown of Tgbr3 and suppress TGF- $\beta$ pathway activity in $368 \mathrm{~T} 1$ and 482N1 cells. Cells were infected with shRNAs as in e and Western analysis was performed for Tgfbr3, pSmad2 and total Smad2 (Smad2). g, Tgfbr3 depletion reduces anchorage-independent growth of 368T1 wt and ATG wt and 482N1 cells. Cells were infected with the listed shRNAs and plated for anchorage-independent growth and colonies were counted as above. Values are technical triplicates, have been performed independently three times, and represent mean $+/-$ s.d. ***: $p<0.0005 ; * *: p<0.005 ; *$ : $\mathrm{p}<0.05$. 
a

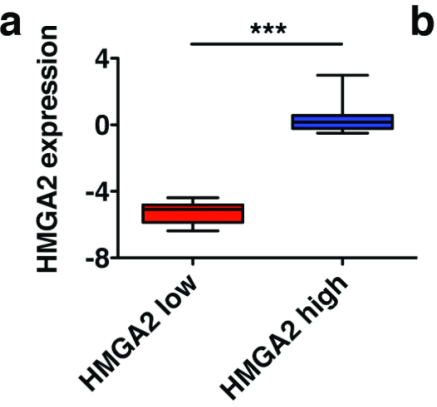

C

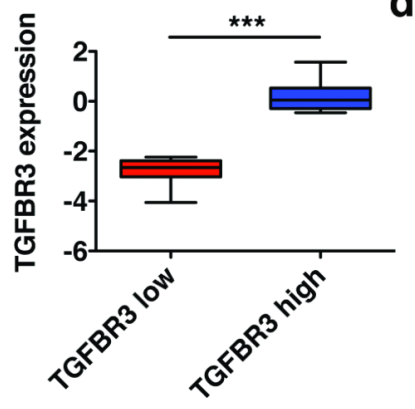

e

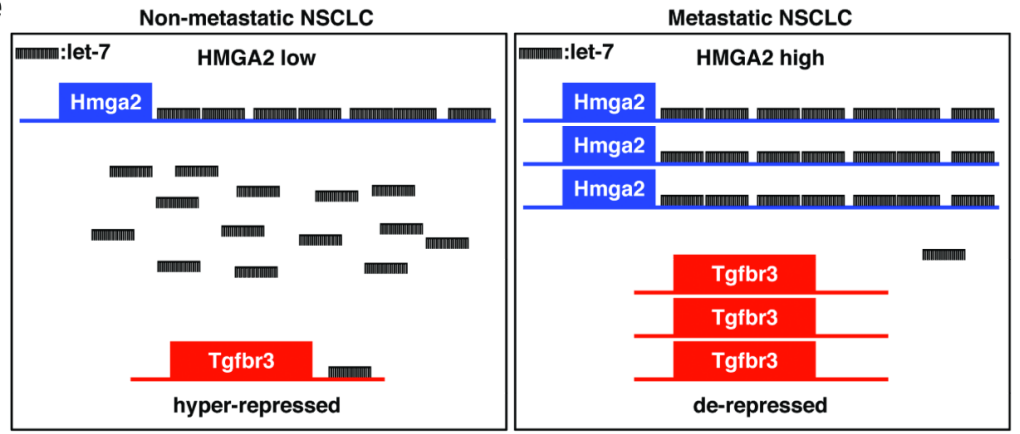

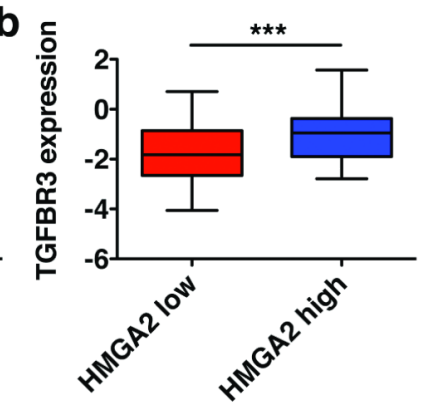

d

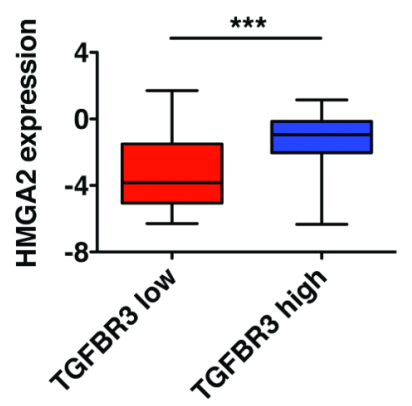

Figure 4. HMGA2 and TGFBR3 are reciprocally and co-ordinately upregulated in NSCLC patients

a, The Cancer Genome Atlas (TCGA) NSCLC gene expression data set was sorted on $H M G A 2$ expression. The top and bottom quartiles (HMGA2 low and high, respectively) were selected (45 patients per group) and $H M G A 2$ expression was compared by box and whisker plot. b. The TCGA data set was sorted into top and bottom quartiles of $H M G A 2$ expression as in a, and TGFBR3 expression was compared by box and whisker plot. c, The TCGA data set was sorted into top and bottom quartiles of TGFBR3 expression (TGFBR3 low and high, respectively) as in a, and TGFBR3 expression was compared by box and whisker plot. d, The TCGA data set was sorted into top and bottom quartiles of TGFBR3 expression as in c, and HMGA2 expression was compared by box and whisker plot. In all box and whisker plots, values are presented on a $\log _{2}$ scale. Significance was assessed by the Mann-Whitney test with a Bonferroni correction for multiple hypothesis testing. ***: p $<0.0005$. e, Model for $\mathrm{Hmga} 2$ acting as a competing endogenous RNA for Tgfbr3. In nonmetastatic NSCLC, Hmga 2 expression is low, leading to suppressed Tgfbr3 expression by let-7. In metastatic NSCLC, Hmga2 expression is elevated, titrating away let-7 from $T g f b r 3$ and allowing for its over-expression. This titration occurs without changes in let-7 expression, reflecting competition for microRNA occupancy by target transcripts. 